\title{
Update to the AWED (Applying Wolbachia to Eliminate Dengue) trial study protocol: a cluster randomised controlled trial in Yogyakarta, Indonesia
}

Katherine L. Anders ${ }^{1 *}$ (D), Citra Indriani ${ }^{2}$, Riris Andono Ahmad², Warsito Tantowijoyo ${ }^{3}$, Eggi Arguni ${ }^{4}$, Bekti Andari ${ }^{5}$, Nicholas P. Jewell ${ }^{6}$, Suzanne M. Dufault ${ }^{7}$, Peter A. Ryan ${ }^{1}$, Stephanie K. Tanamas ${ }^{1}$, Edwige Rancès ${ }^{1}$, Scott L. O’Neill', Cameron P. Simmons ${ }^{1}$ and Adi Utarini ${ }^{8}$

\begin{abstract}
Background: The AWED (Applying Wolbachia to Eliminate Dengue) trial is a parallel, two-arm, non-blinded cluster randomised controlled trial that is under way in Yogyakarta, Indonesia, with the aim of measuring the efficacy of Wolbachia-infected Aedes aegypti deployments in reducing dengue incidence in an endemic setting. Enrolment began in January 2018 and is ongoing. The original study protocol was published in April 2018. Here, we describe amendments that have been made to the study protocol since commencement of the trial.

Methods: The key protocol amendments are (1) a revised study duration with planned end of participant enrolment in August 2020, (2) the addition of new secondary objectives (i) to estimate serotype-specific efficacy of the Wolbachia intervention and (ii) to compare Ae. aegypti abundance in intervention versus untreated clusters, (3) an additional exposure classification for the per-protocol analysis where the Wolbachia exposure index is calculated using only the cluster-level Wolbachia prevalence in the participant's cluster of residence, (4) power re-estimation using a multinomial sampling method that better accounts for randomness in sampling, and (5) the addition of two trial stopping rules to address the potential for persistently low rates of virologically confirmed dengue case enrolment and Wolbachia contamination into untreated clusters. Additional minor changes to the protocol are also described.
\end{abstract}

Discussion: The findings from this study will provide the first experimental evidence for the efficacy of Wolbachia in reducing dengue incidence. Enrolment in the trial will conclude this year (2020) and results will be reported shortly thereafter.

Trial registration: ClinicalTrials.gov, identifier: NCT03055585. Registered on 14 February 2017. Last updated 22 March 2020.

Keywords: Wolbachia, Dengue, Chikungunya, Zika, Vector-borne disease, Cluster randomised trial, Testnegative design, Indonesia

*Correspondence: katie.anders@worldmosquito.org

${ }^{1}$ Institute of Vector Borne Disease, Monash University, 12 Innovation Walk, Melbourne 3800, Victoria, Australia

Full list of author information is available at the end of the article

C The Author(s). 2020 Open Access This article is licensed under a Creative Commons Attribution 4.0 International License, which permits use, sharing, adaptation, distribution and reproduction in any medium or format, as long as you give appropriate credit to the original author(s) and the source, provide a link to the Creative Commons licence, and indicate if changes were made. The images or other third party material in this article are included in the article's Creative Commons licence, unless indicated otherwise in a credit line to the material. If material is not included in the article's Creative Commons licence and your intended use is not permitted by statutory regulation or exceeds the permitted use, you will need to obtain permission directly from the copyright holder. To view a copy of this licence, visit http://creativecommons.org/licenses/by/4.0/. The Creative Commons Public Domain Dedication waiver (http://creativecommons.org/publicdomain/zero/1.0/) applies to the data made available in this article, unless otherwise stated in a credit line to the data. 


\section{Update}

This update relates to the study protocol for a cluster randomised controlled trial to evaluate the efficacy of Wolbachia-infected mosquito deployments to reduce dengue incidence in Yogyakarta, Indonesia: the Applying Wolbachia to Eliminate Dengue (AWED) trial. This update should be read in conjunction with the original protocol publication [1]. The trial registration record on ClinicalTrials.gov has been updated to reflect these protocol amendments.

\section{Study duration}

A 12-month extension to the trial duration was approved by the independent data monitoring committee (IDMC) to account for lower-than-expected dengue incidence in Yogyakarta (and elsewhere in Indonesia) during the first year of the study period. Participant enrolment is now planned to conclude on 31 August 2020 unless early trial termination occurs because of Wolbachia contamination into untreated clusters or consistently low enrolment rates of virologically confirmed dengue (VCD) cases (see 'Trial stopping rules' below).

\section{Secondary endpoints}

\section{DENV serotype-specific efficacy}

The primary endpoint of the trial includes virologically confirmed dengue virus (DENV) infections of any (or unknown) serotype combined, based on the detection of DENV RNA in a pan-dengue reverse transcription polymerase chain reaction (RT-PCR) or detection of non-structural protein 1 (NS1) antigen. We now make explicit the use of a second serotype-specific RT-PCR to determine the infecting DENV serotype in samples positive in the pan-dengue PCR and to estimate serotype-specific efficacy of the Wolbachia intervention. In laboratory experiments, the degree to which Wolbachia reduces the DENV transmission potential of Aedes aegypti is dependent on the infecting virus serotype, and DENV1 transmission is least affected [2]. A secondary analysis will estimate the serotype-specific efficacy of Wolbachia deployments in reducing symptomatic dengue virus infection with a known infecting serotype, for each of the four serotypes in turn or as many as are detected in the study population. The same intention-to-treat and per-protocol analyses described for the primary endpoint will be used here. Case populations will be restricted to each of the DENV serotypes in turn, and the same control population will be used as for analysis of the primary endpoint.

\section{Zika and chikungunya}

If at least 20 virologically confirmed Zika or chikungunya cases are detected, a secondary analysis will estimate the efficacy of Wolbachia deployments in reducing the incidence of symptomatic virologically confirmed Zika virus and chikungunya virus infection. No formal analysis will be undertaken if fewer than 20 virologically confirmed cases of Zika or chikungunya are detected. Only a descriptive analysis of the temporal and spatial distribution of cases will be carried out.

\section{Notified dengue cases}

A proposed method for statistical analysis was added to the secondary endpoint of assessing the impact of Wolbachia deployment on routine dengue case notifications. An interrupted time series analysis of monthly dengue haemorrhagic fever (DHF) notifications by kelurahan, before and after Wolbachia releases, will be used to evaluate the impact of Wolbachia deployment on DHF case notifications. Methods will be developed and validated a priori to classify area-level Wolbachia exposure status in a way that aligns with the administrative (kelurahan) boundaries by which dengue cases are reported. A separate statistical analysis plan will be developed for this endpoint, and the results will be reported in a secondary publication subsequent to the publication of the main trial results.

\section{Aedes species abundance}

The AWED trial provides an opportunity to explore whether fitness costs associated with Wolbachia infection of Ae. aegypti that have been identified in laboratory environments (e.g., fecundity and egg survivorship) manifest as a lower population size of adult mosquitoes in areas where Wolbachia is established versus untreated areas. A secondary endpoint was added to measure and compare the population size of adult mosquitoes in Wolbachia-treated versus untreated clusters using existing data from BG trap mosquito collections. Poisson regression will be used to test the null hypothesis of no difference in the abundance of Ae. aegypti and other species by treatment arm, incorporating BG trap as a random effect to account for the clustered sampling of mosquitoes by BG trap.

\section{Prevalence of arbovirus-infected Aedes aegypti mosquitoes}

Assessing the impact of Wolbachia deployment on the prevalence of arbovirus-infected Ae. aegypti mosquitoes will no longer be carried out. Findings so far indicate that the overall prevalence of dengue virus-infected $A e$. aegypti mosquitoes is too low for this secondary objective to be feasible within the resources available.

\section{Clinical sampling procedures}

In a situation where a consenting participant has already had blood collected for clinical investigations on the day of enrolment, a second blood sample will not be collected for 
research purposes. Rather, the residual blood sample will be retained and used for study investigations. Two implications of this are noted: (1) the sample volume may be lower than the usual $3 \mathrm{~mL}$, and (2) informed consent may be obtained from the participant after the clinical blood sampling has occurred (but before retrieval of the residual sample for use in the study). This amendment was made in response to a number of participants who were enrolled without a blood sample, and thus no classifiable diagnostic results to determine case/control status, due to their refusal to have two blood samples collected in one visit, one each for clinical diagnostic purposes and for our study.

\section{Diagnostic algorithm}

Previously, only samples that were PCR-negative for dengue, chikungunya and Zika were subsequently tested by DENV NS1 enzyme-linked immunosorbent assay (ELISA). All samples will now be tested by both RT-PCR and NS1 ELISA (Bio-Rad dengue NS1 Platelia ELISA, Bio-Rad Laboratories, Hercules, CA, USA) (Fig. 1). All samples positive for DENV in the triplex reverse transcription polymerase chain reaction (RT-PCR) will be tested in a serotypespecific RT-PCR to determine the infecting serotype.

\section{Per-protocol analysis}

\section{Dataset for analysis}

The same participant dataset will now be used for the intention-to-treat analysis and the per-protocol analysis (i.e., including all participants recruited after Wolbachia is considered established in the intervention area, defined as 1 month after completion of releases in the last cluster).

\section{Alternative exposure classification}

An additional per-protocol analysis will be conducted in which the Wolbachia exposure index (WEI) is calculated using only the cluster-level Wolbachia prevalence in the participant's cluster of residence (in the month of participant enrolment), ignoring the participant's recent travel history. This recognises that dengue exposure risk may be higher at home versus other locations, rather than assuming an even distribution of exposure risk across daytime hours and locations visited.

\section{Revised statistical methods}

A mixed-effects logistic regression model will be fitted, incorporating time as a random effect and with another random effect for cluster membership. Such models yield an estimate, and associated confidence interval, for the relative risk. The WEI strata will first be included as an ordinal covariate, and the slope of the WEI variable will be tested for a difference from zero. The WEI strata will additionally be included as a nominal (unordered) covariate to calculate stratum-specific incidence rate ratios (relative to the baseline $0-0.2$ stratum). This will allow examination of a 'dose response' relationship. An additional benefit of transforming WEI to a categorical

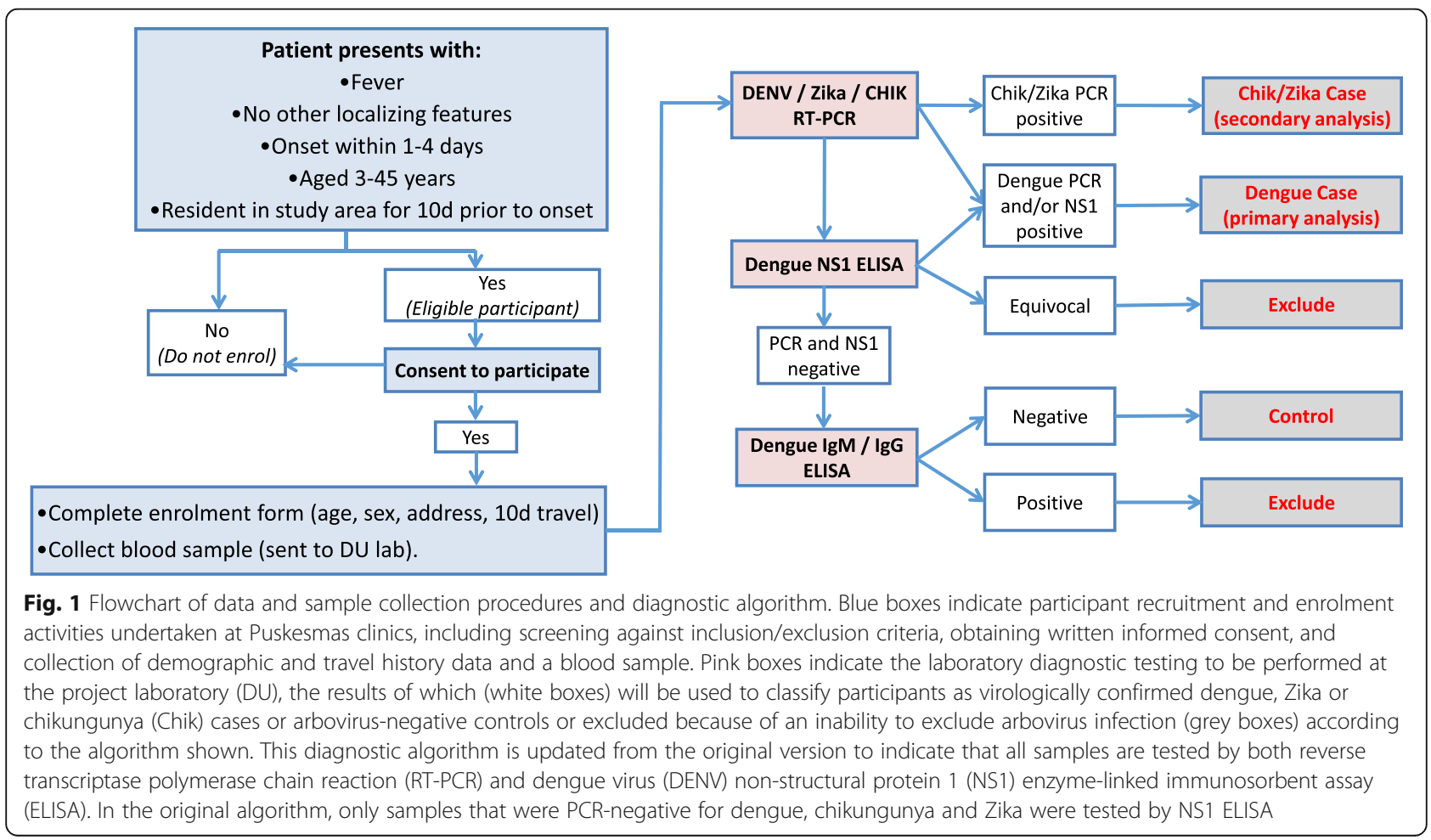


variable is that it avoids any assumption of linearity in the dose response relationship.

\section{Power calculations}

Statistical power for the trial was re-estimated in January 2019 using a multinomial simulation method, with a range of sample sizes and effect sizes, as compared with the original power estimation which used a deterministic simulation of a range of effect sizes but with a fixed sample size. This revised method better accounts for randomness in sampling, including variability in the distribution of dengue cases and non-dengue febrile patients between clusters. This indicates that adequate power $(\geq 80 \%)$ can be achieved with a substantially lower sample size than the 1000 dengue cases assumed for the original power simulation scenario. There is more than $80 \%$ power to detect a risk ratio (RR) of 0.5 (or smaller) with a sample of $400 \mathrm{VCD}$ cases and $4 \times 400$ test-negative controls.

Additional simulations in September 2019 explored the potential impact on power if a number of untreated clusters are 'lost' to Wolbachia contamination. Given 400 enrolled VCD cases and a true effect size of $50 \%$ $(R R=0.5)$, contamination of three or six untreated clusters is expected to result in about $7 \%$ and about $14 \%$ loss of statistical power, respectively.

\section{Interim analysis}

An interim analysis was originally planned for the midpoint of the study (i.e., after enrolment of 500 dengue cases from an initial target sample size of 1000). The reestimation of statistical power described above indicates that the trial will be adequately powered even with a smaller sample size, and the threshold of 500 cases is unlikely to be reached. The multinomial sampling method used in the power re-estimation means that it is no longer necessary to re-calculate sample size using the observed inter-cluster distribution of participants, as originally stated. In November 2019, the IDMC advised that no interim analysis was required.

\section{Trial stopping rules}

Additional criteria for early termination of the trial were introduced in October 2019 in response to increasing Wolbachia contamination in several untreated clusters. The first rule addresses the possibility that a power loss due to contamination may compromise the intention-to-treat analysis and would see the trial stop if five or more untreated clusters are classified as contaminated. A cluster will be defined as contaminated when the cluster-level Wolbachia frequency is more than $50 \%$ for two monthly monitoring events within a 6-month rolling window and more than $50 \%$ of the BG traps in the cluster have detected Wolbachia during those monitoring events.
The second rule addresses the potential for consistently low rates of VCD case enrolment to make continued recruitment until August 2020 futile in terms of increasing sample size (statistical power) and would see the trial stop if five or fewer VCD cases are enrolled in any 3-month rolling window (commencing 1 November 2019).

An assessment of both the Wolbachia monitoring results and accrual of VCD cases will be made each month. The final decision to terminate or modify the study continues to rest with the trial steering committee (TSC). The participant dataset for analysis will include all those cases enrolled up until the end of the calendar month in which the stopping rule was triggered, even if the date the TSC endorses the decision to stop the trial falls in the following month.

\section{Trial status}

Recruitment into the trial began in January 2018 and is ongoing, and completion is expected by August 2020. The current approved protocol is version 5.1 approved by Universitas Gadjah Mada (UGM) ethics committee on 22 January 2020 and Monash University ethics committee on 31 January 2020. This update includes additional minor amendments made in protocol version 6.0, which was under institutional review board review in March 2020.

\section{Abbreviations}

AWED: Applying Wolbachia to Eliminate Dengue; DENV: Dengue virus; DHF: Dengue haemorrhagic fever; ELISA: Enzyme-linked immunosorbent assay; IDMC: Independent data monitoring committee; NS1: Non-structural protein 1; PCR: Polymerase chain reaction; RR: Risk ratio; RT-PCR: Reverse transcriptase polymerase chain reaction; TSC: Trial steering committee; VCD: Virologically confirmed dengue; WEl: Wolbachia exposure index

\section{Acknowledgements}

The authors acknowledge all members of the World Mosquito Program (WMP)-Indonesia and WMP-Global teams who have contributed to the implementation of the AWED trial.

\section{Authors' contributions}

KLA, RAA, CI, WT, NPJ, SKT, CPS and AU conceived the protocol changes reported here. SMD performed the revision to the power calculation and simulation of the impact on power of Wolbachia contamination into untreated clusters. All authors read and approved the submitted manuscript.

\section{Funding}

This study is funded by Yayasan Tahija (the Tahija Foundation), Indonesia. The funders have not had a role in the design of the study and will not be involved in the collection, analysis or interpretation of the data.

\section{Availability of data and materials}

The full trial protocol will be made publicly available at the time of publication of trial results. The datasets generated in this study will be made available upon reasonable request to the corresponding author.

\section{Ethics approval and consent to participate}

This trial protocol has been approved by the Universitas Gadjah Mada (UGM) ethics committee (approval number KE/FK/105/EC/2016) and Monash University Human Research Ethics Committee (approval number 0960). Written informed consent will be obtained from participants (or their guardian where the participant is a minor). In addition, participants between 
13 and 17 years of age will be invited to sign a consent form indicating that they understand the research and agree to participate.

\section{Consent for publication}

Not applicable.

\section{Competing interests}

The authors declare that they have no competing interests.

\section{Author details}

${ }^{1}$ Institute of Vector Borne Disease, Monash University, 12 Innovation Walk, Melbourne 3800, Victoria, Australia. ${ }^{2}$ Department of Biostatistics, Epidemiology and Population Health and Centre for Tropical Medicine, Faculty of Medicine, Universitas Gadjah Mada, Jl. Medika, Yogyakarta 55281, Indonesia. ${ }^{3}$ World Mosquito Program, Centre for Tropical Medicine, Faculty of Medicine, Universitas Gadjah Mada, Jl. Medika, Yogyakarta 55281, Indonesia. ${ }^{4}$ Department of Pediatrics and Centre for Tropical Medicine, Faculty of Medicine, Universitas Gadjah Mada, Jl. Medika, Yogyakarta 55281, Indonesia. ${ }^{5}$ Centre for Tropical Medicine, Faculty of Medicine, Universitas Gadjah Mada, Jl. Medika, Yogyakarta 55281, Indonesia. ${ }^{6}$ Centre for Statistical Methodology, London School of Hygiene and Tropical Medicine, Keppel St, London WC1E 7HT, UK. ${ }^{7}$ School of Public Health, University of California, 2121 Berkeley Way, Berkeley 94720-7360, CA, USA. ${ }^{8}$ Department of Health Policy and

Management, and Centre for Tropical Medicine, Faculty of Medicine,

Universitas Gadjah Mada, J. Medika, Yogyakarta 55281, Indonesia.

Received: 29 March 2020 Accepted: 5 May 2020

Published online: 25 May 2020

\section{References}

1. Anders KL, Indriani C, Ahmad RA, Tantowijoyo W, Arguni E, Andari B, et al. The AWED trial (Applying Wolbachia to Eliminate Dengue) to assess the efficacy of Wolbachia-infected mosquito deployments to reduce dengue incidence in Yogyakarta, Indonesia: study protocol for a cluster randomised controlled trial. Trials. 2018;19:302

2. Ferguson NM, Kien DT, Clapham H, Aguas R, Trung VT, Chau TN, et al. Modeling the impact on virus transmission of Wolbachia-mediated blocking of dengue virus infection of Aedes aegypti. Sci Transl Med. 2015;7:279ra37.

\section{Publisher's Note}

Springer Nature remains neutral with regard to jurisdictional claims in published maps and institutional affiliations.

Ready to submit your research? Choose BMC and benefit from:
- fast, convenient online submission
- thorough peer review by experienced researchers in your field
- rapid publication on acceptance
- support for research data, including large and complex data types
- gold Open Access which fosters wider collaboration and increased citations
- maximum visibility for your research: over 100M website views per year
At BMC, research is always in progress.
Learn more biomedcentral.com/submissions

